\title{
Targeting miRNAs for Pancreatic Cancer Therapy
}

\author{
Min Shi ${ }^{a, b}$, Dacheng $\mathrm{Xie}^{\mathrm{c}}$, Yong Gaod, and Keping Xie ${ }^{\mathrm{b},{ }^{*}}$ \\ aDepartment of Surgery, Shanghai Jiaotong University Affiliated Ruijin Hospital, Shanghai, \\ People's Republic of China \\ ${ }^{\mathrm{b}}$ Gastroenterology, Hepatology \& Nutrition, The University of Texas MD Anderson Cancer Center, \\ Houston, TX, USA \\ 'Department of Oncology, Shanghai Jiaotong University Affiliated First People's Hospital, \\ Shanghai, People's Republic of China \\ dDepartment of Oncology and Tumor Institute, Shanghai East Hospital, Tongji University School \\ of Medicine, Shanghai, People's Republic of China
}

\begin{abstract}
Pancreatic cancer (PC) is the fourth leading cause of cancer-related deaths in the United States and has a median 5-year survival rate less than 5\%. Although surgery offers the best chance for a cure for pancreatic cancer, less than $20 \%$ of patients are eligible for potentially curative resection, because in most cases, the cancer has already spread locally or to distant organs at diagnosis, precluding resection. MicroRNAs (miRNAs) are small noncoding, endogenous, single-stranded RNAs that are pivotal regulators of posttranscriptional gene expression. Extensive studies of miRNAs over the past several years have revealed that the expression of miRNAs is frequently deregulated in pancreatic cancer patients and that this deregulation contributes to the pathogenesis and aggressiveness of the disease. Currently, investigators are studying the use of miRNAs as diagnostic and/or prognostic biomarkers and therapeutic tools for pancreatic cancer. Rapid discovery of many miRNA targets and their relevant pathways has contributed to the development of miRNA-based therapeutics. In particular, the transcription factor Forkhead box M1 (FOXM1) is overexpressed in the majority of cancer patients, including those with pancreatic cancer. This overexpression is implicated to have a role in tumorigenesis, progression, and metastasis. This important role of FOXM1 affirms its usefulness in therapeutic interventions for pancreatic cancer. In this review, we summarize the current knowledge and concepts concerning the involvement of miRNAs and FOXM1 in pancreatic cancer development and describe the roles of the miRNAFOXM1 signaling pathway in pancreatic cancer initiation and progression. Additionally, we describe some of the technical challenges in the use of the miRNA-FOXM1 signaling pathway in pancreatic cancer treatment.
\end{abstract}

\section{Keywords}

miRNA; FOXM1; pancreatic cancer; transcription; invasion; metastasis; therapy

\footnotetext{
*Address correspondence to this author at the Department of Gastroenterology, Hepatology \& Nutrition, Unit 1466, The University of Texas MD Anderson Cancer Center, 1515 Holcombe Boulevard, Houston, TX 77030, USA. Phone: 713-794-5073; Fax:

713-745-3654; kepxie@mdanderson.org.
} 


\section{INTRODUCTION}

Pancreatic cancer is the fourth leading cause of cancer-related deaths in industrialized countries. In 2012, physicians diagnosed 43,920 new cases of pancreatic cancer and 37,390 patients died of it in the United States [1]. At present, only about $20 \%$ of pancreatic cancer patients are eligible for potentially curative resection. Even in these patients, the 5-year survival rate rarely exceeds $20 \%$ owing to early relapse or metastasis [2]. The vast majority of pancreatic cancer patients have locally advanced or metastatic disease at diagnosis, both of which are associated with an extremely poor prognosis [3]. Surgical resection followed by a 6-month course of adjuvant gemcitabine-based chemotherapy is the standard treatment of early-stage pancreatic cancer; patients with locally advanced or metastatic disease are candidates for systemic palliative chemotherapy. Even with this treatment, pancreatic cancer patients have a median 5-year survival rate of 5\% and median survival duration of 6 months after diagnosis [1]. Therefore, a novel therapeutic strategy based on improved understanding of the molecular mechanisms underlying pancreatic cancer initiation and progression is urgently needed.

MicroRNAs (miRNAs) are short noncoding RNAs 20-24 nucleotides in length that posttranscriptionally regulate gene expression [4]. They guide the binding of the RNAinduced silencing complex to regions of partial complementarity located mainly within $3^{\prime}$ untranslated regions in target mRNA, resulting in mRNA degradation and/or translational inhibition [5]. Importantly, miRNAs play critical roles in a broad range of biological processes, including proliferation, differentiation, apoptosis, and stress response, linking them with numerous human diseases, including cancer [6].

Genome-wide gene expression profiling of cancer has independently and consistently identified Forkhead box protein M1 (FOXM1) as one of the most commonly upregulated genes in human solid tumors, including liver, prostate, brain, breast, lung, colon, pancreatic, skin, cervical, ovarian, mouth, blood, and central nervous system cancers [7-9]. In addition, FOXM1 is implicated to have a role in tumor cell proliferation, migration, invasion, angiogenesis, and metastasis. This important role of FOXM1 in cancer affirms its usefulness in therapeutic intervention for the disease [10].

In this review, we briefly describe miRNAs and FOXM1 and their roles in pancreatic cancer development and progression as well as the possibility of modulating miRNA and FOXM1 functions for therapeutic gain. Furthermore, we highlight the significance of miRNAFOXM1 signaling in pancreatic cancer development and progression, which may be a preventive and therapeutic strategy for pancreatic cancer.

\section{MIRNAS IN PANCREATIC CANCER}

\section{Biogenesis, Function, and Targets of miRNAs}

MicroRNAs (miRNAs) are short non-coding RNAs of 20-24 nucleotides in length [4]. More than 1500 mature human miRNA sequences are listed in the miRBase database (www.mirbase.org), and these sequences play important roles in virtually all biological 
pathways in mammals and other multicellular organisms [11,12]. The miRNA maturation process includes several posttranscriptional processing steps [13,14] (Fig. 1). For most miRNAs, transcription is carried out by RNA polymerase II, yielding a $5^{\prime}$-capped, $3^{\prime}$ polyadenylated primary miRNA (pri-miRNA) that can be longer than 1000 bases and contain a single or multiple hairpin stem-loop structures (miRNA clusters) with mature miRNAs on one arm of these stem loops [13]. Following transcription, the pri-miRNA is processed by the nuclear RNase III endonuclease DROSHA, yielding a 60- to 70-nucleotide double-stranded hairpin structure, precursor miRNA (premiRNA) [15]. In turn, pre-miRNA is actively exported to the cytoplasm via the Exportin-5 complex, where it is further processed by the RNase III endonuclease DICER. This enzyme cleaves pre-miRNA at the opposite end from Drosha near the terminal loop of the pre-miRNA hairpin, leading to the production of an approximately 22-nucleotide double-stranded molecule-miRNA duplex $[16,17]$. This duplex is then incorporated into the miRNA-induced silencing complex (miRISC) [18], where it is unwound, facilitating the separation of the mature antisense miRNA (guide strand) and transfer of it to Argonaute proteins within the miRISC [17]. Meanwhile, the passenger strand is released and usually rapidly degraded. Following complex miRISC assembly, the mature single-stranded miRNA guides the miRISC to target mRNAs [19]. Target recognition and hybridization in animals occur primarily because of incomplete base-pairing complementarity between the miRNA and target mRNA, resulting in mismatches and bulges that lead to target gene silencing, which can occur via translational repression and/or mRNA degradation. In the rare cases of nearly perfect miRNA complementarity with the target mRNA in animals, the mRNA can be endonucleolytically cleaved. Whether translational repression occurs at or after the initiation of translation is unclear [20]. In addition, degradation of the target mRNA induced by miRNA without cleavage by Argonaute may lead to deadenylation, decapping, and exonucleolytic degradation of mRNA [4,5].

\section{Roles of miRNAs in Pancreatic Cancer}

MiRNA expression profiles in pancreatic cancer cells are different from those in normal pancreatic cells and pancreatic cells in patients with chronic pancreatitis. Most miRNA expression profile analyses have demonstrated that miRNA expression is downregulated in pancreatic tumors but upregulated in the normal pancreas and that the expression pattern is tissue specific (Table 1) [21-24].

Intraductal papillary mucinous neoplasms (IPMNs) are noninvasive precursor lesions for pancreatic cancer. The miRNAs miR-155 (mean, 11.6-fold) and miR-21 (mean, 12.1-fold) have exhibited the highest relative fold-changes in expression in these precursor lesions. Using locked nucleic acid in situ hybridization, researchers identified the expression of miR-155 in 53 of $64(83 \%)$ IPMNs but only 4 of $54(7 \%)$ normal pancreatic ducts and of miR-21 in 52 of $64(81 \%)$ IPMNs but only 1 of $54(2 \%)$ normal pancreatic ducts (P < 0.0001 ), indicating that aberrant miRNA expression is an early event in the multistage progression of pancreatic cancer and that miR-155 and miR-21 may be biomarkers for IPMNs in clinical specimens of pancreatic cancer [25]. 
MiRNAs are implicated to have roles in cancer initiation and progression because of their ability to affect the expression of genes and proteins that regulate cell proliferation and/or death. Nagao et al. [26] analyzed the miRNA expression profiles of five pancreatic ductal adenocarcinomas and five normal pancreatic tissue specimens using a microarray platform. They found that the expression of 28 miRNAs was higher in the adenocarcinomas than in the normal pancreatic tissue, whereas that of 48 miRNAs was lower in the tumors. MiR-21 was the most highly overexpressed miRNA; it was also highly expressed in $75 \%$ of 65 pancreatic ductal adenocarcinomas examined using real-time polymerase chain reaction. High miR-21 expression was correlated with poor prognosis in the pancreatic ductal adenocarcinoma patients $(P=0.045)$. Furthermore, the immunohistochemical patterns of expression of the tumor suppressors programmed cell death 4 (PDCD4) and tissue inhibitor of metalloproteinase (TIMP3) were significantly associated with both upregulated expression of miR-21 (both $\mathrm{P}<0.05$ ) and poor survival durations $(\mathrm{P}<0.001$ and $\mathrm{P}=0.001$, respectively). This suggests that miR-21 overexpression is associated with the biological behavior of pancreatic ductal adenocarcinoma via downregulation of PDCD4 and TIMP3 expression, resulting in tumor progression and the adverse clinical course of this cancer. In another microarray analysis, the expression of miR-372, miR-204, miR-10a, and miR-10b differed by particularly large amounts (>10-fold) in pancreatic cell lines and human pancreatic ductal epithelial (HPDE) cells. Specifically, 13 of 15 pancreatic cancer cell lines had 2.1- to 36.4-fold higher levels of miR-10b expression than did HPDE cells.

Microdissection analysis revealed that miR-10b expression was higher in pancreatic cancer cells $(n=5)$ than in normal pancreatic ductal cells $(n=10 ; P<0.02)$. Analysis of pancreatic tumor specimens demonstrated that high miR-10b expression was associated with a short overall survival duration $(\mathrm{P}=0.014)$. Furthermore, miR-10b expression correlated with the invasiveness of pancreatic cancer cells $(\mathrm{P}<0.01)$ [27].

Hamada et al. [28] conducted a comprehensive analysis of miRNA expression profiles in invasive ductal adenocarcinoma, intraductal papillary mucinous adenoma, intraductal papillary mucinous carcinoma, and human pancreatic cancer cell lines to identify essential miRNAs that regulate invasive growth of pancreatic cancer cells. They detected reduced expression of miR-126 in the invasive ductal adenocarcinoma and pancreatic cancer cell line. They also found that miR-126 targeted disintegrin and metalloproteinase domaincontaining protein 9 (ADAM9), which is highly expressed in pancreatic cancer cells. Overexpression of miR-126 and small interfering RNA (siRNA)-based knockdown of expression of ADAM9 in pancreatic cancer cells resulted in reduced cellular migration and invasion and induction of the epithelial marker E-cadherin. This revealed that the miR-126/ ADAM9 axis plays an essential role in inhibition of invasive growth of pancreatic cancer cells.

Ji et al. [29] demonstrated that restoration of miR-34 expression in pancreatic cancer cells downregulated the expression of B-cell lymphoma-2 and Notch 1/2, which markedly inhibited clonogenic cell growth and invasion and induced apoptosis and cell-cycle arrest at G1 and G2/M phase. Moreover, CD44+/CD133+ MiaPaCa2 cells were enriched with tumorsphere-forming and tumor-initiating cells or cancer stem/progenitor cells with high levels of Notch/B-cell lymphoma-2 expression and loss of miR-34 expression, implying that 
miR-34 plays an important role in pancreatic cancer stem cell self-renewal and/or cell fate determination. In another study, investigators found lower miR-146a expression in pancreatic cancer cells than in normal HPDE cells. Overexpression of miR-146a inhibited the invasive capacity of pancreatic cancer cells with concomitant downregulation of epidermal growth factor receptor and the nuclear factor- $\mathrm{\kappa B}(\mathrm{NF}-\kappa \mathrm{B})$-regulatory kinase interleukin 1 receptor-associated kinase 1 [30]. Ectopic expression of miR-96 through a synthetic miRNA precursor may have inhibited V-Ki-ras2 Kirsten rat sarcoma viral oncogene homolog (KRAS) expression, decreased protein kinase B signaling, and triggered apoptosis in pancreatic cancer cells. In human pancreatic tumor specimens, miR-96 expression was downregulated or deleted when the delection was asssociated with elevated KRAS expression. These two aspects revealed that miR-96 may suppress KRAS and function as a tumor suppressor gene in pancreatic cancer cells [31].

Hao et al. [32] reported that the expression of miR-483-3p was much higher in pancreatic tumors than in paired adjacent normal tissue specimens. Furthermore, deleted in pancreatic cancer 4 (DPC4)/SMAD family member 4 (Smad4) are targets of miR-483-3p, and their expression levels are inversely correlated with each other in human pancreatic tumor specimens. Specifically, ectopic expression of miR-483-3p markedly represses deleted in pancreatic cancer DPC4/Smad4 protein expression in pancreatic cancer cell lines and promotes cell proliferation and colony formation in vitro. MiR-301a may downregulate NF$\kappa \mathrm{B}-$-repressing factor (Nkrf) expression and elevate NF- $\kappa \mathrm{B}$ activation. Researchers found that Nkrf expression was downregulated and miR-301a expression was upregulated in human pancreatic adenocarcinoma cells. Moreover, miR-301a's inhibition or upregulation of Nkrf expression in pancreatic cancer cells may have led to reduced NF- $\kappa \mathrm{B}$ target gene expression and attenuated xenograft tumor growth, indicating that miR-301a overexpression contributes to NF- $\kappa \mathrm{B}$ activation and promotes pancreatic cancer initiation and progression [33]. In another study, miR-148a expression was four fold higher in normal pancreatic ductal cells than in pancreatic cancer cells. Stable lentivirus-mediated overexpression of miR-148a in the pancreatic cancer cell line IMIM-PC2 may have inhibited tumor cell growth and colony formation via targeting of CDC25B [34].

Zhao et al. [35] demonstrated that miR-148b expression was markedly downregulated in 48 pairs of human pancreatic tumor specimens and five pancreatic cancer cell lines.

Furthermore, the downregulated miR-148b expression was correlated with increased tumor size, late TNM stage, lymphatic invasion, distant metastasis, and poor prognosis in pancreatic cancer patients. Overexpression of miR-148b dramatically suppressed the growth of pancreatic cancer cells, which was attributable to induction of apoptosis and cell-cycle arrest at $\mathrm{S}$ phase. Further studies revealed that AMP-activated protein kinase a1 (AMPKa1) may be the direct target gene for miR-148b, and overexpressed AMPKa1 was inversely correlated with miR-148b expression in pancreatic cancer cells [35]. Silencing of AMPKa1 by RNA interference inhibited the growth of pancreatic cancer cells in vitro and in vivo and induced apoptosis and cell-cycle arrest in and inhibited invasion of cancer cells, which are consistent with the effects of miR-148b overexpression. All of these results demonstrated that miR-148b may inhibit pancreatic cancer cell proliferation and invasion and enhance the chemosensitivity of pancreatic cancer by targeting AMPKa1. 
Authors reported that members of the miR-124 family, including miR-124-1, miR-124-2, and miR-124-3, were more highly methylated in pancreatic tumors than in normal pancreatic tissue, indicating that hypermethylation-mediated silencing of miR-124 was a frequent event in the tumors [36]. MiR-124 inhibited cancer cell proliferation, invasion, and metastasis, and downregulation of miR-124 expression was highly associated with poor survival in pancreatic cancer patients. Furthermore, miR-124 directly targeted and downregulated the expression of Ras-related C3 botulinum toxin substrate 1, which may have led to inactivation of the mitogen-activated protein kinase kinase 4/c-Jun N-terminal kinase/c-Jun pathway. All of these findings demonstrated that miR-124 is a tumor suppressor miRNA that is epigenetically silenced in pancreatic cancer cells [36].

\section{FOXM1 EXPRESSION IN PANCREATIC CANCER CELLS}

FOXM1, also known as HFH11, MPP2, WIN, and Trident, belongs to the Forkhead superfamily of transcription factors, which are evolutionarily conserved in the winged helix/ Forkhead DNA-binding domain [37-41]. The human FOXM1 gene consists of 10 exons, two of which-Va (A1) and VIIa (A2) - are alternatively spliced. This splicing gives rise to three distinct FOXM1 isoforms: FOXM1a, FOXM1b, and FOXM1c. FOXM1a harbors Va and VIIa and is transcriptionally inactive owing to disruption of its transactivation domain by VIIa. In comparison, FOXM1b, which contains neither of the two exons, and FOXM1c, which contains only $\mathrm{Va}$, are transcriptionally active and can activate their target gene expression via different mechanisms. Because FOXM1 is a transcription factor essential for expression of many genes key to regulation of multiple aspects of tumor cell survival, growth, epithelial-to-mesenchymal transition (EMT), angiogenesis, and metastasis, abnormal FOXM1 expression may contribute to human cancer development and progression $[42,43]$.

A study demonstrated that FOXM1 protein and mRNA expression levels were higher in pancreatic carcinoma lesions than in paired adjacent noncancerous tissue specimens [44]. A high level of expression of FOXM1 was significantly correlated with clinical stage $(\mathrm{P}=$ 0.004), lymph node metastasis $(\mathrm{P}=0.009)$, and histological differentiation $(\mathrm{P}=0.017)$. Patients with high FOXM1 expression had significantly shorter survival durations than did patients with low FOXM1 expression $(\mathrm{P}<0.001)$. Multivariate analysis revealed that FOXM1 is an independent marker of poor prognosis, indicating that FOXM1 can be used as prognostic and therapeutic molecular marker for pancreatic cancer [44].

Researchers have shown that FOXM1 plays important roles in regulation of the expression of genes involved in cell proliferation, differentiation, and transformation. For example, FOXM1 is overexpressed in a variety of aggressive human carcinomas, including pancreatic carcinoma. Wang et al. [45] found that downregulation of FOXM1 expression inhibited the growth and decreased the migration and invasion of pancreatic cancer cells. FOXM1 downregulation also decreased cell populations in the S phase. FOXM1 siRNA-transfected cells had lower expression of cyclin B, cyclin D1, and cyclin-dependent kinase 2 than did control cells, whereas the transfected cells had higher expression of $\mathrm{p} 21^{\mathrm{Cip} 1}$ and $\mathrm{p} 27^{\mathrm{Kip} 1}$. Furthermore, downregulation of FOXM1 expression reduced matrix metalloproteinase (MMP)-2, MMP-9, and vascular endothelial growth factor expression, resulting in inhibition 
of migration, invasion, and angiogenesis. This suggested that downregulation of FOXM1 expression is a novel approach to inhibition of pancreatic cancer progression.

Investigators found that treatment with genistein was responsible for lowering the rate of pancreatic cancer progression. Genistein inhibited cell growth and induced apoptosis and concomitantly attenuated FOXM1 and its downstream genes, such as survivin, cdc25a, MMP-9, and vascular endothelial growth factor, resulting in inhibition of pancreatic cancer cell invasion. Downregulation of FOXM1 expression by siRNA prior to genistein-based treatment resulted in enhanced inhibition of growth and induction of apoptosis. This study demonstrated the molecular role of FOXM1 in mediating the biological effects of genistein in pancreatic cancer cells, suggesting that FOXM1 is a novel target for the treatment of pancreatic cancer [46].

EMT, a process by which epithelial cells acquire mesenchymal characteristics, leading to increased migratory and invasive potential of cancer cells, has a critical role in metastasis [47]. Overexpression of FOXM1 may lead to the acquisition of the EMT phenotype via activation of mesenchymal cell markers, zinc finger E-box-binding homeobox 1 (ZEB1), zinc finger E-box-binding homeobox 2 (ZEB2), Snail2, E-cadherin, and vimentin in pancreatic cancer cells [48]. Also, FOXM1 promotes EMT, migration, invasion, and metastasis in pancreatic tumors via direct transcriptional activation of caveolin-1, a key structural protein in the caveolae that is strongly implicated to play a role in the development of cancer [49].

Kong et al. [50] identified five FOXM1 isoforms in pancreatic cancer cells: FOXM1a, FOXM1b, and FOXM1c along with two isoforms tentatively designated as FOXM1b1 and FOXM1b2 because they are closely related to FOXM1b. FOXM1c was predominantly expressed in pancreatic tumors and cancer cell lines, whereas FOXM1a expression was generally undetectable in them. Functional analysis revealed that FOXM1b, FOXM1b1, FOXM1b2, and FOXM1c but not FOXM1a promoted pancreatic tumor growth and metastasis. Consistently, FOXM1b, FOXM1b1, FOXM1b2, and FOXM1c activated transcription of their specific downstream genes. This study demonstrated that overexpression of specific FOXM1 isoforms critically regulates pancreatic cancer development and progression by enhancing tumor cell invasion and metastasis and that targeting of specific FOXM1 isoforms effectively attenuates pancreatic cancer development and progression.

\section{FOXM1-MEDIATED TARGETING OF MIRNAS FOR PANCREATIC CANCER THERAPY}

\section{The miRNA-FOXM1 Signaling Pathway in Pancreatic Cancer Cells}

Given the importance of miRNA and FOXM1 expression in cancer initiation and progression, modulation of the miRNA and FOXM1 signaling pathways may play a critical role in cancer biology, so investigation of it is urgently needed. Researchers found that in pancreatic cancer patients, overexpression of FOXM1 led to acquisition of an EMT phenotype via activation of the mesenchymal cell markers ZEB1, ZEB2, Snail2, and vimentin, which is consistent with increased sphere-forming (pancreatospheres) capacity 
and expression of the cancer stem cell surface markers CD44 and EpCAM [48].

Furthermore, overexpression of FOXM1 led to decreased expression of miRNAs (let-7a, let-7b, let-7c, miR-200b, and miR-200c). However, re-expression of miR-200b inhibited the expression of ZEB1, ZEB2, vimentin, and FOXM1 and reduced the expression of Ecadherin, leading to reversal of the EMT phenotype. This demonstrated that FOXM1 overexpression is responsible for acquisition of the EMT and cancer stem cell phenotypes, which is mediated in part the regulation of miR-200b expression [48]. Authors have reported involvement of the miRNA-FOXM1 signaling pathway in tumors other than pancreatic cancer. For example, the expression of has-miR-370 decreased in human gastric tumors, and expression of FOXM1 was directly downregulated by has-miR-370 in gastric cancer cell lines. Helicobacter pylori and cytotoxin-associated gene A inhibited has-miR-370 expression, which led to FOXM1 overexpression and cell proliferation [51]. In NSCLC cases, investigators found that miR-134 expression was correlated with invasive potential and EMT phenotype and identified FOXM1, a potential metastasis promoter, as a direct and functional target of miR-134, demonstrating that miR-134 inhibited EMT via FOXM1 [52]. In another study, downregulation of miR-370 expression was a frequent event in leukemia cell lines and primary leukemic cells obtained from patients with de novo acute myeloid leukemia. In addition, ectopic expression of miR-370 in HL60 and K562 cells led to cellgrowth arrest and senescence. In contrast, downregulation of miR-370 expression enhanced the proliferation of the leukemic cells. Mechanistically, miR-370 may function as a tumor suppressor by targeting FOXM1; epigenetic silencing of miR-370 thus leads to upregulation of FOXM1 expression and consequently contributes to acute myeoloid leukemia development and progression [53].

\section{FOXM1-Mediated Targeting of miRNAs As a Therapeutic Strategy for Pancreatic Cancer}

The oncogenic transcription factor FOXM1 is overexpressed in the majority of human cancers and implicated to have roles in cell migration, invasion, angiogenesis, and metastasis. The important role of FOXM1 in cancer development affirms its usefulness in therapeutic interventions for cancer [54]. Recently reported data suggest that targeting FOXM1 in monotherapy or combination therapy for cancer is beneficial [10]. After being extensively demonstrated to be deregulated in cancer cells, researchers are now studying miRNAs as targets and tools for cancer treatment. Downregulation of miRNA expression to normal levels holds remarkable potential for these therapeutic interventions $[55,56]$.

MiRNAs are applied to cancer therapy using two systems. In one system, miRNA antagonists such as anti-miRs, locked nucleic acids, and antagomiRs are used to inhibit the expression of oncogenic miRNAs. MiRNA antagonists are single-stranded RNA molecules about 21-23 nucleotides in length and complementary to mature target miRNAs. They specifically silence the expression of miRNA and then upregulate the expression of hundreds of genes predicted to be repressed by downmodulated expression of miRNA. In the other system, a tumor suppressor miRNA mimic is reintroduced into the cells to restore loss of function of the target genes [57].

The miRNA delivery method is the most significant problem in miRNA-based therapy. In 2007, Valadi et al. [58] found that exosomes contain miRNAs. They showed that miRNAs are not only intracellular gene regulators but also humoral factors, suggesting that miRNAs 
act as tools for cell-cell communication. Johnstone and colleagues discovered exosomes in 1987 when they observed that during maturation, sheep reticulocytes secreted exosomes to remove the transferring receptors [59]. Exosomes are homogenous in shape, range from 50 to $100 \mathrm{~nm}$ in diameter, and float on a sucrose gradient at a density of $1.13-1.19 \mathrm{~g} / \mathrm{ml}$ [60]. They are formed via invagination of the plasma membrane into endosomes followed by inward vesiculation and vesicular release into the extracellular space [61,62]. Exosomes can be identified by their enrichment in Alix, tumor susceptibility gene 101, heat shock protein 70, and the tetraspanins CD63, CD81, and CD9 [62,63]. Exosome secretion occurs in most cell types under both physiological and pathological conditions, especially tumor cells and hematopoietic cells, including reticulocytes [64-67], dendritic cells [68], B and T lymphocytes [69-71], platelets [72], mast cells [73,74], macrophages [75], epithelial cells [76], fibroblasts [77], astrocytes, and neurons [78]. The extent of exosome secretion can be affected not only by the Ca2+ level [79-81] but also by amino acid availability, as nutrient starvation, stress, rapamycin, and hormone therapy induce autophagy, thus blocking exosome secretion [82].

As described above, resolving the problem of miRNA delivery is essential for the use of miRNAs in cancer treatment. Therefore, examination of exosomal miRNAs in cancer treatment is natural. Exosomal miR-223 in tumor-associated macrophages is transported to breast cancer cells, supporting the idea that macrophages regulate the invasiveness of breast cancer cells via exosome-mediated delivery of oncogenic miRNAs [83]. In addition, exosomal miRNAs derived from hepatocellular carcinoma cells can be taken up by other cells and target transforming growth factor- $\beta$-activated kinase 1 , resulting in enhancement of transformed cell growth in recipient cells [84]. Investigators also showed that proliferation of a prostate carcinoma cell line was inhibited by the addition of the exosome fraction isolated from a noncancerous prostate epithelial cell line. These observations suggested that exosomal miRNAs derived from noncancerous cells were transferred to cancerous cells and inhibited the proliferation of the latter. Indeed, expression of some tumor-suppressive miRNAs, such as miR-16, miR-205, and miR-143, was downregulated in prostate cancer cell lines at the cellular and extracellular levels. This supports the idea that secretory tumor-suppressive miRNAs are transferred from noncancerous to cancerous cells in accordance with the miRNA concentration gradient.

To examine the contribution of secretory tumor-suppressive miRNAs to cancer initiation in depth, researchers generated miR-143-overproducing HEK293 cells and observed that this prostate cancer cell line had an approximately $50 \%$ decrease in proliferation via suppression of expression of the miR-143 target gene KRAS after the addition of an exosome derived from the miR-143-overproducing HEK293 cells. Importantly, the decreased proliferation was reversed by transfection of anti-miR-143 of an antisense oligonucleotide specifically targeting miR-143 in the prostate cancer cell line. These data indicate that the cell-growth inhibition was attributable to the secretory miR-143 contained in the exosome [85]. In addition, exogenously transduced miR-143 did not suppress the proliferation of noncancerous cells, suggesting that excessive numbers of tumor-suppressive miRNAs did not impose an additional growth-inhibitory effect on normal cells, in which expression of tumor-suppressive miRNAs is maintained at physiological levels. Furthermore, the researchers observed no overt side effects on exosome-mediated gene delivery by a dendritic 
cell-derived exosome in vivo. These studies indicate that miRNAs that specifically target FOXM1 can be loaded into exosomes and then delivered to cancer cells and thus may be developed as feasible anticancer drugs (Fig. 2).

\section{CONCLUSIONS AND PERSPECTIVES}

MiRNAs are well known to be key players in a wide variety of biological processes, including tumor initiation, proliferation, apoptosis, invasion, metastasis, and prognosis [86]. Like other malignant tumors, pancreatic cancer has unique miRNA expression patterns that differentiate normal pancreatic tissue from benign inflammatory pancreatic tissue and pancreatic tumors [87]. Identification of unique patterns of deregulated miRNA expression in pancreatic cancer cells provides valuable information that may serve as a molecular biomarker for tumor diagnosis, identification of low- and high-risk patients, disease prognosis, cancer prevention, and prediction of cancer therapeutic responses [88].

Originally, FOXM1 caught the attention of investigators as a critical regulator of cell proliferation and cell-cycle progression [10]. Increased understanding of FOXM1 function and how it is regulated will undoubtedly provide clues for deciphering the mechanisms of tumorigenesis and cancer progression. All of the findings from relevant recent studies point to the fact that FOXM1 may hold the key to significant progress in cancer treatment. For example, FOXM1 is a potential therapeutic target for cancer, as its expression is upregulated in numerous human malignancies, but it is not expressed in nondividing normal cells, thus indicating specificity for cancer cells in a targeted therapeutic approach [89].

The discovery of miRNAs and advancement of research methods have led to an enormous number of questions concerning details about miRNA expression regulation and posttranscriptional regulation regarding their roles in the signaling pathways for cell proliferation, apoptosis, and invasion as well as improvement of research methods. The most promising field in cancer research is the delineation of tumorigenic pathways and use of cancer therapeutics [90]. The miRNA-FOXM1 signaling pathway may have a promising role in inhibition of cancer initiation and progression, and miRNAs that specifically target FOXM1 can be used as anticancer drugs. Two aspects of the potential use of FOXM1targeting miRNA as anti-cancer drugs should be specifically considered: identification of miRNAs that can effectively inhibit FOXM1 expression and development of simple, efficient methods of delivering miRNAs to cancer cells.

\section{Acknowledgments}

This work was supported in part by grants R01-CA129956, R01-CA148954, R01CA152309, and R01CA172233 from the National Institutes of Health (to Keping Xie, MD, PhD) and Shanghai Key Special Department \#ZK2012A26, and \#1281272917 and \#81172022 from the Natural Science Foundation of China (to Yong Gao, $\mathrm{MD}, \mathrm{PhD}$ ).

\section{REFERENCES}

[1]. Siegel R, Naishadham D, Jemal A. Cancer statistics, 2012. CA Cancer J Clin. 2012; 62:10-29. [PubMed: 22237781]

[2]. Paulson AS, Tran Cao HS, Tempero MA, Lowy AM. Therapeutic advances in pancreatic cancer. Gastroenterology. 2013; 144:1316-26. [PubMed: 23622141] 
[3]. Stathis A, Moore MJ. Advanced pancreatic carcinoma: current treatment and future challenges. Nat Rev Clin Oncol. 2010; 7:163-72. [PubMed: 20101258]

[4]. Kim VN, Han J, Siomi MC. Biogenesis of small RNAs in animals. Nat Rev Mol Cell Biol. 2009; 10:126-39. [PubMed: 19165215]

[5]. Bartel DP. MicroRNAs: target recognition and regulatory functions. Cell. 2009; 136:215-33. [PubMed: 19167326]

[6]. Lujambio A, Lowe SW. The microcosmos of cancer. Nature. 2012; 482:347-55. [PubMed: 22337054]

[7]. Pilarsky C, Wenzig M, Specht T, Saeger HD, Grutzmann R. Identification and validation of commonly overexpressed genes in solid tumors by comparison of microarray data. Neoplasia. 2004; 6:744-50. [PubMed: 15720800]

[8]. Uddin S, Ahmed M, Hussain A, et al. Genome-wide expression analysis of Middle Eastern colorectal cancer reveals FOXM1 as a novel target for cancer therapy. Am J Pathol. 2011; 178:537-47. [PubMed: 21281787]

[9]. Okabe H, Satoh S, Kato T, et al. Genome-wide analysis of gene expression in human hepatocellular carcinomas using cDNA microarray: identification of genes involved in viral carcinogenesis and tumor progression. Cancer Res. 2001; 61:2129-37. [PubMed: 11280777]

[10]. Halasi M, Gartel AL. Targeting FOXM1 in cancer. Biochem Pharmacol. 2013; 85:644-52. [PubMed: 23103567]

[11]. Griffiths-Jones S, Grocock RJ, van Dongen S, Bateman A, Enright AJ. miRBase: microRNA sequences, targets and gene nomenclature. Nucleic Acids Res. 2006; 34:D140-4. [PubMed: 16381832]

[12]. Griffiths-Jones S, Saini HK, van Dongen S, Enright AJ. miRBase: tools for microRNA genomics. Nucleic Acids Res. 2008; 36:D154-8. [PubMed: 17991681]

[13]. Bentwich I, Avniel A, Karov Y, et al. Identification of hundreds of conserved and nonconserved human microRNAs. Nat Genet. 2005; 37:766-70. [PubMed: 15965474]

[14]. Berezikov E, van Tetering G, Verheul M, et al. Many novel mammalian microRNA candidates identified by extensive cloning and RAKE analysis. Genome Res. 2006; 16:1289-98. [PubMed: 16954537]

[15]. Krol J, Loedige I, Filipowicz W. The widespread regulation of microRNA biogenesis, function and decay. Nat Rev Genet. 2010; 11:597-610. [PubMed: 20661255]

[16]. Kent OA, Mendell JT. A small piece in the cancer puzzle: microRNAs as tumor suppressors and oncogenes. Oncogene. 2006; 25:6188-96. [PubMed: 17028598]

[17]. Kim VN. MicroRNA biogenesis: coordinated cropping and dicing. Nat Rev Mol Cell Biol. 2005; 6:376-85. [PubMed: 15852042]

[18]. Tang G. siRNA and miRNA: an insight into RISCs. Trends Biochem Sci. 2005; 30:106-14. [PubMed: 15691656]

[19]. Huang JC, Babak T, Corson TW, et al. Using expression profiling data to identify human microRNA targets. Nat Methods. 2007; 4:1045-9. [PubMed: 18026111]

[20]. Ambros V. The functions of animal microRNAs. Nature. 2004; 431:350-5. [PubMed: 15372042]

[21]. Bloomston M, Frankel WL, Petrocca F, et al. MicroRNA expression patterns to differentiate pancreatic adenocarcinoma from normal pancreas and chronic pancreatitis. JAMA. 2007; 297:1901-8. [PubMed: 17473300]

[22]. Lee EJ, Gusev Y, Jiang J, et al. Expression profiling identifies microRNA signature in pancreatic cancer. Int J Cancer. 2007; 120:1046-54. [PubMed: 17149698]

[23]. Szafranska AE, Davison TS, John J, et al. MicroRNA expression alterations are linked to tumorigenesis and non-neoplastic processes in pancreatic ductal adenocarcinoma. Oncogene. 2007; 26:4442-52. [PubMed: 17237814]

[24]. Zhang Y, Li M, Wang H, et al. Profiling of 95 microRNAs in pancreatic cancer cell lines and surgical specimens by real-time PCR analysis. World J Surg. 2009; 33:698-709. [PubMed: 19030927]

[25]. Habbe N, Koorstra JB, Mendell JT, et al. MicroRNA miR-155 is a biomarker of early pancreatic neoplasia. Cancer Biol Ther. 2009; 8:340-6. [PubMed: 19106647] 
[26]. Nagao Y, Hisaoka M, Matsuyama A, et al. Association of microRNA-21 expression with its targets, PDCD4 and TIMP3, in pancreatic ductal adenocarcinoma. Mod Pathol. 2012; 25:112-21. [PubMed: 21983937]

[27]. Nakata K, Ohuchida K, Mizumoto K, et al. MicroRNA-10b is overexpressed in pancreatic cancer, promotes its invasiveness, and correlates with a poor prognosis. Surgery. 2011; 150:91622. [PubMed: 22018284]

[28]. Hamada S, Satoh K, Fujibuchi W, et al. MiR-126 acts as a tumor suppressor in pancreatic cancer cells via the regulation of ADAM9. Mol Cancer Res. 2012; 10:3-10. [PubMed: 22064652]

[29]. Ji Q, Hao X, Zhang M, et al. MicroRNA miR-34 inhibits human pancreatic cancer tumorinitiating cells. PLoS One. 2009; 4:e6816. [PubMed: 19714243]

[30]. Li Y, Vandenboom TG 2nd, Wang Z, et al. miR-146a suppresses invasion of pancreatic cancer cells. Cancer Res. 2010; 70:1486-95. [PubMed: 20124483]

[31]. Yu S, Lu Z, Liu C, et al. miRNA-96 suppresses KRAS and functions as a tumor suppressor gene in pancreatic cancer. Cancer Res. 2010; 70:6015-25. [PubMed: 20610624]

[32]. Hao J, Zhang S, Zhou Y, Hu X, Shao C. MicroRNA 483-3p suppresses the expression of DPC4/ Smad4 in pancreatic cancer. FEBS Lett. 2011; 585:207-13. [PubMed: 21112326]

[33]. Lu Z, Li Y, Takwi A, et al. miR-301a as an NF-kappaB activator in pancreatic cancer cells. EMBO J. 2011; 30:57-67. [PubMed: 21113131]

[34]. Liffers ST, Munding JB, Vogt M, et al. MicroRNA-148a is down-regulated in human pancreatic ductal adenocarcinomas and regulates cell survival by targeting CDC25B. Lab Invest. 2011; 91:1472-9. [PubMed: 21709669]

[35]. Zhao G, Zhang JG, Liu Y, et al. miR-148b functions as a tumor suppressor in pancreatic cancer by targeting AMPKalpha1. Mol Cancer Ther. 2013; 12:83-93. [PubMed: 23171948]

[36]. Wang P, Chen L, Zhang J, et al. Methylation-mediated silencing of the miR-124 genes facilitates pancreatic cancer progression and metastasis by targeting Rac1. Oncogene. Jan 21.2013 doi: 10.1038/onc.2012.598. [Epub ahead of print].

[37]. Korver W, Roose J, Heinen K, et al. The human TRIDENT/HFH-11/FKHL16 gene: structure, localization, and promoter characterization. Genomics. 1997; 46:435-42. [PubMed: 9441747]

[38]. Laoukili J, Stahl M, Medema RH. FoxM1: at the crossroads of ageing and cancer. Biochim Biophys Acta. 2007; 1775:92-102. [PubMed: 17014965]

[39]. Kaestner KH, Lee KH, Schlondorff J, Hiemisch H, Monaghan AP, Schutz G. Six members of the mouse forkhead gene family are developmentally regulated. Proc Natl Acad Sci U S A. 1993; 90:7628-31. [PubMed: 7689224]

[40]. Clark KL, Halay ED, Lai E, Burley SK. Co-crystal structure of the HNF-3/fork head DNArecognition motif resembles histone H5. Nature. 1993; 364:412-20. [PubMed: 8332212]

[41]. Clevidence DE, Overdier DG, Tao W, et al. Identification of nine tissue-specific transcription factors of the hepatocyte nuclear factor 3/forkhead DNA-binding-domain family. Proc Natl Acad Sci U S A. 1993; 90:3948-52. [PubMed: 7683413]

[42]. Weigel D, Jurgens G, Kuttner F, Seifert E, Jackle H. The homeotic gene fork head encodes a nuclear protein and is expressed in the terminal regions of the Drosophila embryo. Cell. 1989; 57:645-58. [PubMed: 2566386]

[43]. Ye H, Kelly TF, Samadani U, et al. Hepatocyte nuclear factor 3/fork head homolog 11 is expressed in proliferating epithelial and mesenchymal cells of embryonic and adult tissues. Mol Cell Biol. 1997; 17:1626-41. [PubMed: 9032290]

[44]. Xia JT, Wang H, Liang LJ, et al. Overexpression of FOXM1 is associated with poor prognosis and clinicopathologic stage of pancreatic ductal adenocarcinoma. Pancreas. 2012; 41:629-35. [PubMed: 22249132]

[45]. Wang Z, Banerjee S, Kong D, Li Y, Sarkar FH. Down-regulation of Forkhead Box M1 transcription factor leads to the inhibition of invasion and angiogenesis of pancreatic cancer cells. Cancer Res. 2007; 67:8293-300. [PubMed: 17804744]

[46]. Wang Z, Ahmad A, Banerjee S, et al. FoxM1 is a novel target of a natural agent in pancreatic cancer. Pharm Res. 2010; 27:1159-68. [PubMed: 20354770]

[47]. Hanahan D, Weinberg RA. Hallmarks of cancer: the next generation. Cell. 2011; 144:646-74. [PubMed: 21376230] 
[48]. Bao B, Wang Z, Ali S, et al. Over-expression of FoxM1 leads to epithelial-mesenchymal transition and cancer stem cell phenotype in pancreatic cancer cells. J Cell Biochem. 2011; 112:2296-306. [PubMed: 21503965]

[49]. Huang C, Qiu Z, Wang L, et al. A novel FoxM1-caveolin signaling pathway promotes pancreatic cancer invasion and metastasis. Cancer Res. 2012; 72:655-65. [PubMed: 22194465]

[50]. Kong X, Li L, Li Z, et al. Dysregulated Expression of FOXM1 Isoforms Drives Progression of Pancreatic Cancer. Cancer Res. 2013; 73:3987-96. [PubMed: 23598278]

[51]. Feng Y, Wang L, Zeng J, et al. Fork head box M1 is overexpressed in Helicobacter pyloriinduced gastric carcinogenesis and is negatively regulated by hsa-miR-370. Mol Cancer Res. 2013; 11:834-44. [PubMed: 23576572]

[52]. Li J, Wang Y, Luo J, et al. miR-134 inhibits epithelial to mesenchymal transition by targeting FOXM1 in non-small cell lung cancer cells. FEBS Lett. 2012; 586:3761-5. [PubMed: 23010597]

[53]. Zhang X, Zeng J, Zhou M, et al. The tumor suppressive role of miRNA-370 by targeting FoxM1 in acute myeloid leukemia. Mol Cancer. 2012; 11:56. [PubMed: 22900969]

[54]. Wang Z, Ahmad A, Li Y, Banerjee S, Kong D, Sarkar FH. Forkhead box M1 transcription factor: a novel target for cancer therapy. Cancer Treat Rev. 2010; 36:151-6. [PubMed: 20022709]

[55]. Borralho PM, Kren BT, Castro RE, da Silva IB, Steer CJ, Rodrigues CM. MicroRNA-143 reduces viability and increases sensitivity to 5-fluorouracil in HCT116 human colorectal cancer cells. FEBS J. 2009; 276:6689-700. [PubMed: 19843160]

[56]. Borralho PM, Simoes AE, Gomes SE, et al. miR-143 overexpression impairs growth of human colon carcinoma xenografts in mice with induction of apoptosis and inhibition of proliferation. PLoS One. 2011; 6:e23787. [PubMed: 21901135]

[57]. Bader AG, Brown D, Winkler M. The promise of microRNA replacement therapy. Cancer Res. 2010; 70:7027-30. [PubMed: 20807816]

[58]. Valadi H, Ekstrom K, Bossios A, Sjostrand M, Lee JJ, Lotvall JO. Exosome-mediated transfer of mRNAs and microRNAs is a novel mechanism of genetic exchange between cells. Nat Cell Biol. 2007; 9:654-9. [PubMed: 17486113]

[59]. Johnstone RM, Adam M, Hammond JR, Orr L, Turbide C. Vesicle formation during reticulocyte maturation. Association of plasma membrane activities with released vesicles (exosomes). J Biol Chem. 1987; 262:9412-20. [PubMed: 3597417]

[60]. Thery C, Ostrowski M, Segura E. Membrane vesicles as conveyors of immune responses. Nat Rev Immunol. 2009; 9:581-93. [PubMed: 19498381]

[61]. Bedoui S, Prato S, Mintern J, et al. Characterization of an immediate splenic precursor of CD8+ dendritic cells capable of inducing antiviral T cell responses. J Immunol. 2009; 182:4200-7. [PubMed: 19299718]

[62]. Mathivanan S, Ji H, Simpson RJ. Exosomes: extracellular organelles important in intercellular communication. J Proteomics. 2010; 73:1907-20. [PubMed: 20601276]

[63]. Cocucci E, Racchetti G, Meldolesi J. Shedding microvesicles: artefacts no more. Trends Cell Biol. 2009; 19:43-51. [PubMed: 19144520]

[64]. Johnstone RM, Bianchini A, Teng K. Reticulocyte maturation and exosome release: transferrin receptor containing exosomes shows multiple plasma membrane functions. Blood. 1989; 74:1844-51. [PubMed: 2790208]

[65]. Pan BT, Teng K, Wu C, Adam M, Johnstone RM. Electron microscopic evidence for externalization of the transferrin receptor in vesicular form in sheep reticulocytes. J Cell Biol. 1985; 101:942-8. [PubMed: 2993317]

[66]. Fader CM, Savina A, Sanchez D, Colombo MI. Exosome secretion and red cell maturation: Exploring molecular components involved in the docking and fusion of multivesicular bodies in K562 cells. Blood Cells Mol Dis. 2005; 35:153-7. [PubMed: 16099697]

[67]. Geminard C, de Gassart A, Vidal M. Reticulocyte maturation: mitoptosis and exosome release. Biocell. 2002; 26:205-15. [PubMed: 12240554]

[68]. Zitvogel L, Regnault A, Lozier A, et al. Eradication of established murine tumors using a novel cell-free vaccine: dendritic cell-derived exosomes. Nat Med. 1998; 4:594-600. [PubMed: 9585234] 
[69]. Raposo G, Nijman HW, Stoorvogel W, et al. B lymphocytes secrete antigen-presenting vesicles. J Exp Med. 1996; 183:1161-72. [PubMed: 8642258]

[70]. Clayton A, Turkes A, Navabi H, Mason MD, Tabi Z. Induction of heat shock proteins in B-cell exosomes. J Cell Sci. 2005; 118:3631-8. [PubMed: 16046478]

[71]. Peters PJ, Geuze HJ, Van der Donk HA, et al. Molecules relevant for T cell-target cell interaction are present in cytolytic granules of human T lymphocytes. Eur J Immunol. 1989; 19:1469-75. [PubMed: 2789142]

[72]. Heijnen HF, Schiel AE, Fijnheer R, Geuze HJ, Sixma JJ. Activated platelets release two types of membrane vesicles: microvesicles by surface shedding and exosomes derived from exocytosis of multivesicular bodies and alpha-granules. Blood. 1999; 94:3791-9. [PubMed: 10572093]

[73]. Skokos D, Botros HG, Demeure C, et al. Mast cell-derived exosomes induce phenotypic and functional maturation of dendritic cells and elicit specific immune responses in vivo. J Immunol. 2003; 170:3037-45. [PubMed: 12626558]

[74]. Raposo G, Tenza D, Mecheri S, Peronet R, Bonnerot C, Desaymard C. Accumulation of major histocompatibility complex class II molecules in mast cell secretory granules and their release upon degranulation. Mol Biol Cell. 1997; 8:2631-45. [PubMed: 9398681]

[75]. Bhatnagar S, Shinagawa K, Castellino FJ, Schorey JS. Exosomes released from macrophages infected with intracellular pathogens stimulate a proinflammatory response in vitro and in vivo. Blood. 2007; 110:3234-44. [PubMed: 17666571]

[76]. van Niel G, Raposo G, Candalh C, et al. Intestinal epithelial cells secrete exosome-like vesicles. Gastroenterology. 2001; 121:337-49. [PubMed: 11487543]

[77]. Lespagnol A, Duflaut D, Beekman C, et al. Exosome secretion, including the DNA damageinduced p53-dependent secretory pathway, is severely compromised in TSAP6/Steap3-null mice. Cell Death Differ. 2008; 15:1723-33. [PubMed: 18617898]

[78]. Faure J, Lachenal G, Court M, et al. Exosomes are released by cultured cortical neurones. Mol Cell Neurosci. 2006; 31:642-8. [PubMed: 16446100]

[79]. Savina A, Furlan M, Vidal M, Colombo MI. Exosome release is regulated by a calciumdependent mechanism in K562 cells. J Biol Chem. 2003; 278:20083-90. [PubMed: 12639953]

[80]. Merendino AM, Bucchieri F, Campanella C, et al. Hsp60 is actively secreted by human tumor cells. PLoS One. 2010; 5:e9247. [PubMed: 20169074]

[81]. Fader CM, Sanchez D, Furlan M, Colombo MI. Induction of autophagy promotes fusion of multivesicular bodies with autophagic vacuoles in k562 cells. Traffic. 2008; 9:230-50. [PubMed: 17999726]

[82]. Fader CM, Colombo MI. Autophagy and multivesicular bodies: two closely related partners. Cell Death Differ. 2009; 16:70-8. [PubMed: 19008921]

[83]. Yang M, Chen J, Su F, et al. Microvesicles secreted by macrophages shuttle invasionpotentiating microRNAs into breast cancer cells. Mol Cancer. 2011; 10:117. [PubMed: 21939504]

[84]. Kogure T, Lin WL, Yan IK, Braconi C, Patel T. Intercellular nanovesicle-mediated microRNA transfer: a mechanism of environmental modulation of hepatocellular cancer cell growth. Hepatology. 2011; 54:1237-48. [PubMed: 21721029]

[85]. Kosaka N, Iguchi H, Yoshioka Y, Hagiwara K, Takeshita F, Ochiya T. Competitive interactions of cancer cells and normal cells via secretory microRNAs. J Biol Chem. 2012; 287:1397-405. [PubMed: 22123823]

[86]. Du Y, Liu M, Gao J, Li Z. Aberrant MicroRNAs Expression Patterns in Pancreatic Cancer and Their Clinical Translation. Cancer Biother Radiopharm. 2013; 28:361-9. [PubMed: 23621126]

[87]. Park JY, Helm J, Coppola D, Kim D, Malafa M, Kim SJ. MicroRNAs in pancreatic ductal adenocarcinoma. World J Gastroenterol. 2011; 17:817-27. [PubMed: 21412491]

[88]. Rachagani S, Kumar S, Batra SK. MicroRNA in pancreatic cancer: pathological, diagnostic and therapeutic implications. Cancer Lett. 2010; 292:8-16. [PubMed: 20004512]

[89]. Koo CY, Muir KW, Lam EW. FOXM1: from cancer initiation to progression and treatment. Biochim Biophys Acta. 2012; 1819:28-37. [PubMed: 21978825] 
[90]. Papaconstantinou IG, Lykoudis PM, Gazouli M, Manta A, Polymeneas G, Voros D. A review on the role of microRNA in biology, diagnosis, and treatment of pancreatic adenocarcinoma. Pancreas. 2012; 41:671-7. [PubMed: 22695087]

[91]. Torrisani J, Bournet B, du Rieu MC, et al. let-7 MicroRNA transfer in pancreatic cancer-derived cells inhibits in vitro cell proliferation but fails to alter tumor progression. Hum Gene Ther. 2009; 20:831-44. [PubMed: 19323605]

[92]. Szafranska AE, Doleshal M, Edmunds HS, et al. Analysis of microRNAs in pancreatic fineneedle aspirates can classify benign and malignant tissues. Clin Chem. 2008; 54:1716-24. [PubMed: 18719196]

[93]. Chang TC, Wentzel EA, Kent OA, et al. Transactivation of miR-34a by p53 broadly influences gene expression and promotes apoptosis. Mol Cell. 2007; 26:745-52. [PubMed: 17540599]

[94]. Lodygin D, Tarasov V, Epanchintsev A, et al. Inactivation of miR-34a by aberrant CpG methylation in multiple types of cancer. Cell Cycle. 2008; 7:2591-600. [PubMed: 18719384]

[95]. Moriyama T, Ohuchida K, Mizumoto K, et al. MicroRNA-21 modulates biological functions of pancreatic cancer cells including their proliferation, invasion, and chemoresistance. Mol Cancer Ther. 2009; 8:1067-74. [PubMed: 19435867]

[96]. Dillhoff M, Liu J, Frankel W, Croce C, Bloomston M. MicroRNA-21 is overexpressed in pancreatic cancer and a potential predictor of survival. J Gastrointest Surg. 2008; 12:2171-6. [PubMed: 18642050]

[97]. Roldo C, Missiaglia E, Hagan JP, et al. MicroRNA expression abnormalities in pancreatic endocrine and acinar tumors are associated with distinctive pathologic features and clinical behavior. J Clin Oncol. 2006; 24:4677-84. [PubMed: 16966691]

[98]. Gironella M, Seux M, Xie MJ, et al. Tumor protein 53-induced nuclear protein 1 expression is repressed by miR-155, and its restoration inhibits pancreatic tumor development. Proc Natl Acad Sci U S A. 2007; 104:16170-5. [PubMed: 17911264] 


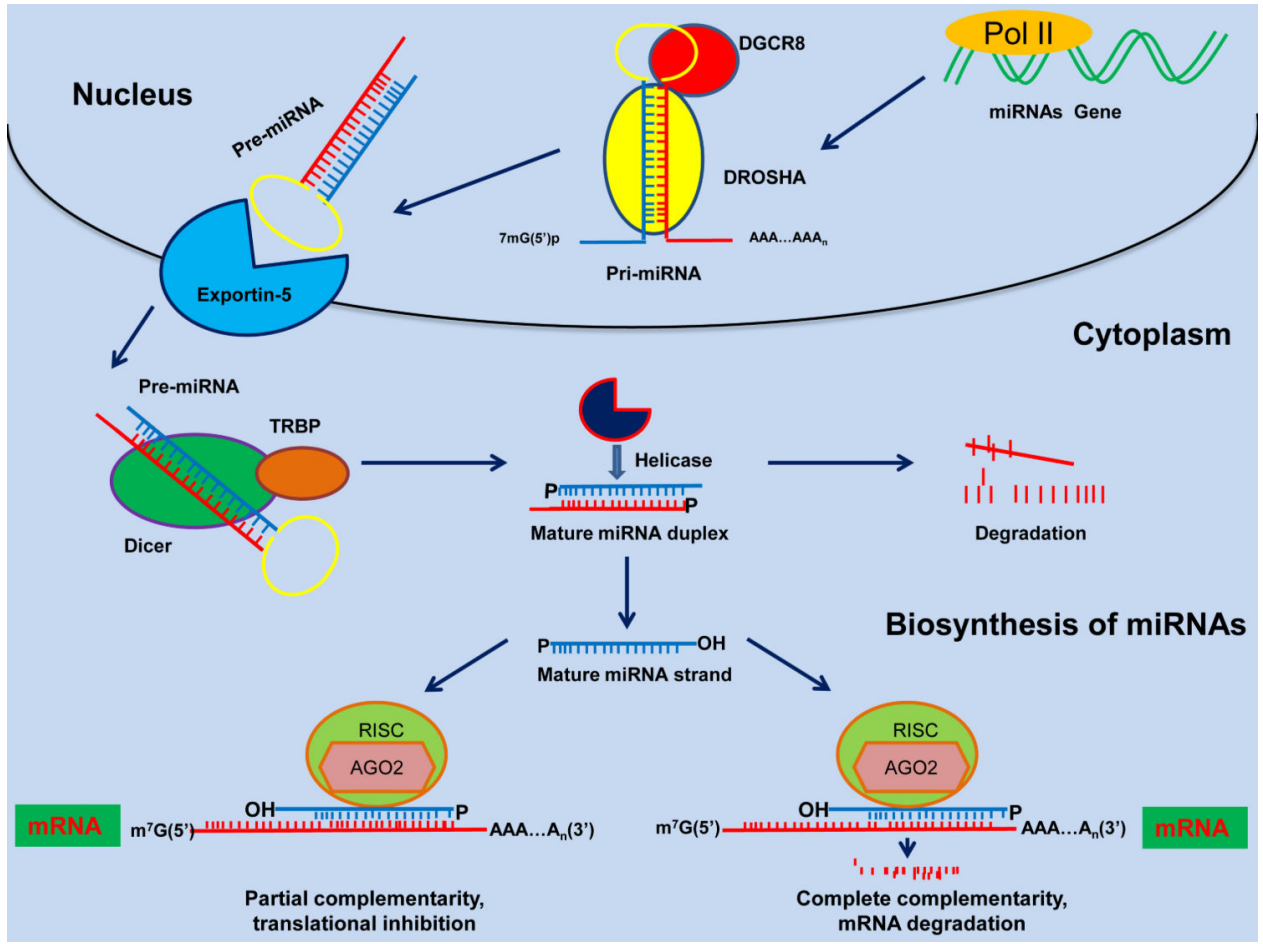

Fig. (1).

The miRNA maturation process. In the nucleus, RNA polymerase II (Pol II) transcribes primiRNA and is cleaved by the DROSHA/DGCR8 complex to form pre-miRNA. The premiRNA is then transported to the cytoplasm by Exportin-5, where it is further processed by the RNase III endonuclease DICER to form mature single-stranded miRNA. Next, the single-stranded miRNA enters the RNA-induced silencing complex (RISC) to suppress expression of the target mRNA via either translational repression (partial complementarity) or target degradation (complete complementarity). 


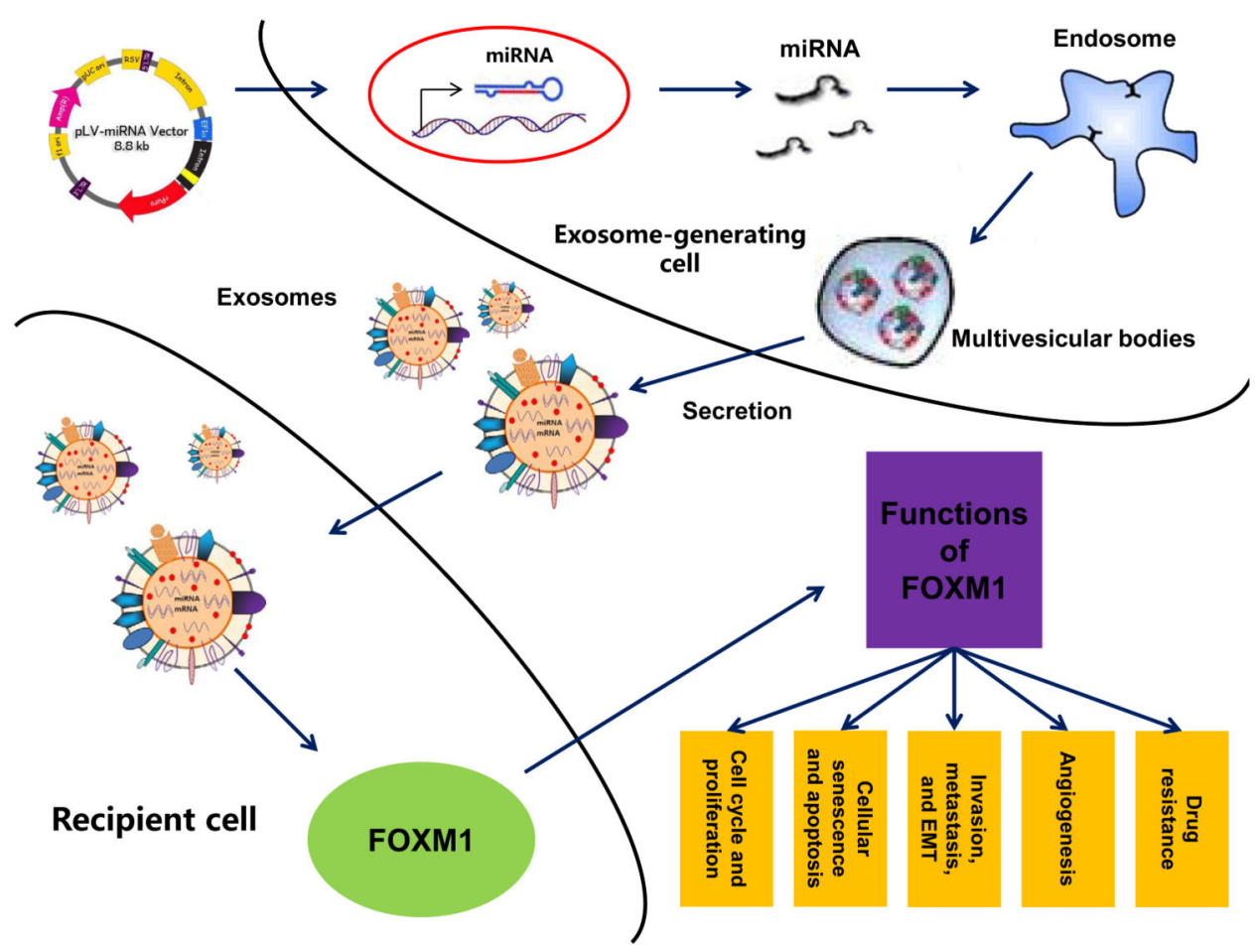

Fig. (2).

Schematic of miRNA transfer by exosomes. MiRNA overexpression is induced in a cell via transfection with an miRNA expression vector. MiRNAs are then selectively incorporated into the intraluminal vesicles of multivesicular bodies (MVBs). Exosomes containing miRNAs are derived from the MVBs and may be released into the extracellular environment via either fusion of MVBs with the cell surface or the budding pathway. Exosomes then may bind to the plasma membrane of a target cell. Recruited exosomes may either fuse directly with the plasma membrane or first be endocytosed and then fuse with the delimiting membrane of an endocytic compartment. Both fusionpathways result in delivery of the exosomal miRNAs to the cytoplasm of the target cell, where it may associate with and silence corresponding mRNA. 
Table 1

Major miRNA Expression Patterns in Pancreatic Cancer Cells

\begin{tabular}{|c|c|c|c|}
\hline MiRNAs & Expression & Target Genes & References \\
\hline let-7 & $\downarrow$ & KRAS and MAPK & [91] \\
\hline $\begin{array}{l}\text { miR-29c, -30a-3p, }-96,-130 b \\
-141,-148 a,-148 b,-216,-217 \\
-375 \text {, and }-494\end{array}$ & $\downarrow$ & $\begin{array}{l}\text { Cyclin-dependent kinase inhibitors 1B }\left(\mathrm{p} 27^{\mathrm{Kip} 1}\right) \text { and } 1 \mathrm{C} \\
\left(\mathrm{p} 57^{\mathrm{Kip} 2}\right)\end{array}$ & {$[23,92]$} \\
\hline miR-34a & $\downarrow$ & CDK6 and $\mathrm{p} 53$ & {$[93,94]$} \\
\hline miR-21 & $\bar{\uparrow}$ & PDCD4 & {$[21,22,95,96]$} \\
\hline $\begin{array}{l}\operatorname{miR}-17,-20,-92-1,-99 a,- \\
99 b,-100,-103,-107,-125 a,- \\
125 b,-130 a,-132,-204,-211, \\
\text { and }-342\end{array}$ & $\uparrow$ & MYC & [97] \\
\hline miR-155 & $\uparrow$ & TP53INP1 & {$[25,98]$} \\
\hline $\begin{array}{l}\operatorname{miR}-31,-18 a,-93,-141,-143, \\
-145,-146 a,-150,-196 a,- \\
196 b,-203,-205,-210,-221,- \\
222,-223 \text {, and }-224\end{array}$ & $\uparrow$ & TIMP3, ZNF462, ZNF563, and STAT3 & {$[23,92]$} \\
\hline $\begin{array}{l}\mathrm{miR}-15 \mathrm{~b},-95,-186,-190,- \\
196-\mathrm{a},-200 \mathrm{~b},-221 \text {, and }-222\end{array}$ & $\uparrow$ & Hoxb8, ZEB1, and SIP1 & {$[24]$} \\
\hline
\end{tabular}

